\title{
Funciones de la documentación informativa en la comunicación externa de los gabinetes de prensa
}

\author{
Juan-Francisco Torregrosa Carmona \\ Universidad Rey Juan Carlos - URJC, España
}

\section{REVIEW}

\begin{abstract}
Resumen
La documentación desempeña un papel clave en el día a día de los gabinetes de comunicación de empresas e instituciones. Más allá de eso, cuando sobrevienen situaciones de crisis, la capacidad de respuesta de la organización depende en buena medida de contar con una adecuada planificación y una gestión documental profesional. Este trabajo repasa las funciones de dicha documentación, plasmadas en los formatos y los cauces habituales de difusión de información tanto a públicos externos, a través de los medios de comunicación, como en el seno de las propias entidades cuya imagen se pretende cuidar. Con el paso de los años la influencia de los gabinetes de prensa dentro de los medios ha ido aumentando, en detrimento de la producción informativa propia. La relativa facilidad con que las empresas e instituciones imponen su discurso mediante notas de prensa y otros recursos amenaza la exclusividad y la calidad de las fuentes en el resultado del trabajo periodístico ofrecido a la ciudadanía.
\end{abstract}

Palabras clave

Documentación ; Gabinetes de comunicación ; Relaciones públicas

\section{Functions of the informative documentation in external communication of the press offices}

\begin{abstract}
The documentation plays a key role day by day in the communication offices of companies and institutions. Beyond it, when situations of crisis occur, the capacity of response of the organization depends of having to a great extensive a suitable planning and a documentary professional management. This piece of work revises the functions of the above mentioned documentation, expressed in the formats and the usual channels of diffusion of information both external spectators, across the mass media, and in the heart of the own entities whose image tries to look care.

Over the years the influence of the press offices within the media has been increasing at the expense of the own informative production. The relative ease with which companies and institutions impose their speech through press releases and other resources threatens the exclusivity and quality of the sources in the result of journalistic work offered to citizens.
\end{abstract}

Keywords

Documentation ; Communication offices ; Public relations

\section{Introducción}

La consideración de las posibilidades y necesidades documentales en los gabinetes de comunicación de las organizaciones implica como necesaria la asunción de una premisa que Piñuel (1997: 22) establece: "Las interacciones comunicativas constituyen la materia prima de la arquitectura organizacional, es decir, de la estructura de relaciones sobre la que reposa la cohesión de posiciones y funciones asignadas a los componentes de una organización: individuos, grupos, medios y procesos de producción, productos obtenidos y reglas generadoras de orden interno y externo; de forma que de la organización se espera siempre un comportamiento que trasciende la individualidad de sus componentes y atañe a un sujeto genérico que actúa en un espacio/tiempo histórico que le es propio a la vida social de las instituciones".

Se denomina gabinete de comunicación "a las fuentes activas, organizadas y habitualmente estables de información que cubren las necesidades comunicativas tanto internas como externas de aquellas organizaciones 
y/o personas de relieve que desean transmitir de sí mismas una imagen positiva a la sociedad influyendo de esta forma en la opinión pública" (Ramírez de la Piscina, 1995: 27). Es deseable que un gabinete de esta naturaleza constituya también dentro de lo posible un centro o servicio de documentación con los objetivos de "evitar costes, duplicaciones de trabajo y, sobre todo, que sea un auténtico profesional el responsable de dicha actividad" (López Yepes, 2011: 169).

Aunque el desarrollo de los últimos decenios haya sido ciertamente exponencial, no podemos caer en la tentación de pensar que la influencia de gobiernos y empresas en la prensa profesional a través de los gabinetes de comunicación y el resto de organizaciones propias del campo de las Relaciones Públicas (en adelante, RR.PP.) sea algo totalmente nuevo. De hecho, salvando las notorias distancias, Maurice Joly, nada menos que en el año 1864, publica una obra titulada Diálogo en el infierno entre Maquiavelo y Montesquieu, en la que el primero le hace la siguiente reflexión al considerado padre de la división de poderes (aunque en realidad perfeccionó la misma teoría anterior expuesta por John Locke): "No os he mostrado todavía más que la parte en cierto modo defensiva del régimen orgánico que impondré a la prensa; ahora os haré ver de qué modo sabré emplear esta institución en provecho de mi poder. Me atrevo a decir que ningún gobierno ha concebido hasta el día de hoy una idea más audaz que la que voy a exponeros. En los países parlamentarios los gobiernos sucumben casi siempre por obra de la prensa; pues bien, vislumbro la posibilidad de neutralizar a la prensa por medio de la prensa misma. Puesto que el periodismo es una fuerza tan poderosa, ¿sabéis qué hará mi gobierno? Se hará periodista, será la encarnación del periodismo" (Joly, 2002: 218). Imposible decirlo mejor.

En la actualidad, la mayoría de los directores de Comunicación (DIRCOM) de las grandes corporaciones ocupa un puesto que tiene la categoría de director general. Como veremos en el esquema siguiente, el organigrama más habitual de la comunicación empresarial e institucional presenta varios niveles:

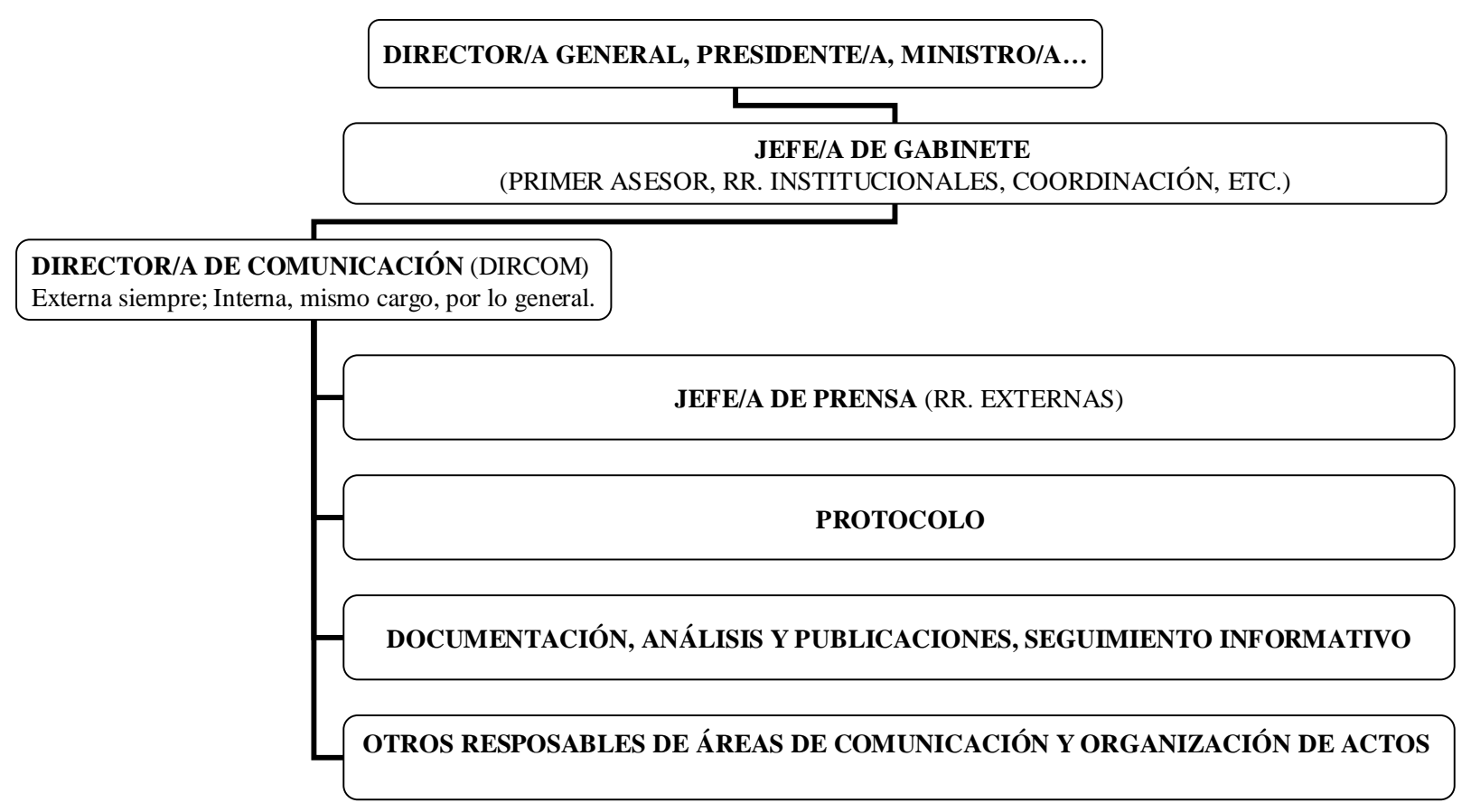

Tabla I. Organigrama de la comunicación empresarial e institucional. Fuente: Elaboración propia.

Martín (2006: 31) define la Comunicación empresarial (corporativa) e institucional como la "creación, coordinación, análisis, desarrollo, difusión y control de toda acción de gestión informativa interna y externa (noticia-actualidad), que diariamente se produce en una empresa o institución tanto a nivel de actividades, servicios o productos, que afecta a un determinado público o colectivo social, que se transmite a través de los medios de comunicación propios y externos, para así potenciar su Imagen corporativa/institucional".

\section{Funciones de la documentación informativa en los gabinetes de prensa}

Conviene, en primer lugar, precisar que la función del trabajo de documentación en el ámbito de la comunicación y el periodismo no ha sido debidamente reconocida. Como destacan García-Gutiérrez y Martínez-Ávila (2014: 495): "El documentalista es un coproductor textual del propio periodista y desvelar tal función históricamente negada contribuiría a la higiene, diversidad y pluralismo de la memoria".

Centrándonos en el trabajo diario actual, bien sea dentro del propio gabinete o dirección de Comunicación y Relaciones Externas (lo más común) o desde un departamento independiente pero relacionado, se trabaja en 
diversas modalidades con la información registrada que la documentación constituye. Lo que se pretende es atender a varias funciones esenciales. Con el fin de dar respuesta a las mismas, se elaboran, analizan, clasifican y/o recuperan documentos de todo tipo (textos, fotografías, diapositivas, carteles, vídeos, grabaciones de audio...).

El adecuado manejo y evaluación de dicha documentación sirve en muchas ocasiones para estrechar el margen de error a la hora de adoptar determinadas decisiones empresariales o institucionales, especialmente en situaciones polémicas o delicadas por cualquier causa. Naturalmente, el manejo profesional de la documentación e información periodística de cada día, así como la emisión de las propias noticias en tanto que fuente para los medios, como es la organización, no constituye la panacea ni la solución milagrosa de nada, pero no es menos cierto que la opinión publicada (que muchos no consideran equiparable a la opinión pública, al menos no necesariamente, o no siempre) ofrece un termómetro que una entidad medianamente relevante no se puede permitir el lujo de obviar. Y de hecho casi ninguna lo hace. Otra cosa será que la estrategia que se adopte sea la públicamente previsible o justo su contraria por motivos de influencia que escapan al conocimiento general. En ocasiones, lo que parece una decisión organizacional disparatada, se torna lógica y comprensible cuando se alcanza a conocer la nueva información o los hechos que en su momento no habían trascendido.

Al igual que en el trabajo periodístico más tradicional, el de los medios, en el ámbito de la gestión informativa de la comunicación empresarial e institucional se cumplen las funciones y los principios de la documentación característicos en la actividad cotidiana de relación con las fuentes de información. Las plataformas de comunicación más recientes, en especial Internet y las redes sociales, conllevan una mayor presencia de las fuentes organizacionales en los medios de comunicación tradicionales, como la prensa, la radio y la televisión. $Y$ es que los gobiernos y las empresas intentan "colocar, vender" sus noticias a los medios conforme a sus muy concretos intereses. Para ello se cuenta, además de con aquellas noticias o hechos por sí mismos relevantes, con exitosos esfuerzos para conseguir la irrupción en la agenda temática de "noticias creadas" (hay muchas formas para lograrlo). Incluso predominan cada vez más, desde hace años, las "no-noticias" (George Hills) o pseudo-acontecimientos políticos, empresariales e institucionales que, pese a ser tales, son cubiertos por todos los medios, en ocasiones con despliegues dignos de mejor causa.

El uso de las fuentes y los recursos documentales está presente, directa o indirectamente, en toda la actividad del gabinete de comunicación, destacadamente los instrumentos de recuperación de la información por excelencia como son las bases de datos. Las de Comunicación es importante actualizarlas con frecuencia, dado que la precariedad y la movilidad alta del sector así lo exige. Deberá haber un suficiente número de campos en cada registro para hacer constar los datos de contacto, la especialidad, observaciones, etcétera. Se suelen emplear desde el programa Access hasta bases de datos especialmente diseñadas.

Otras relevantes funciones habituales de la documentación en los gabinetes de comunicación son:

- Investigación con vistas a la preparación de informes, cronologías, dossieres internos y/o externos.

- Elaboración y difusión de notas de prensa, informes trimestrales o anuales, publicaciones diversas.

- Organización y coordinación de ruedas y conferencias de prensa, actos públicos de empresa o institucionales.

- Entrevistas y otras peticiones de los medios.

- Diseñar, mantener y "alimentar" los sitios web y la Intranet.

- Seguimiento y análisis de la información de la entidad, la competencia más directa y todo el sector en general.

- Archivo documental textual, fotográfico, fonográfico y videográfico de directivos y productos.

- Producción y gestión de múltiples documentos, acciones y soportes para las Relaciones Externas: folletos corporativos, grandes carteles para actos, etcétera. Tramitación de acreditaciones de prensa y permisos administrativos de todas clases (rodajes en parques naturales, cupo de fotógrafos en corridas taurinas...), contratación de azafatas y personal de apoyo, coordinación con otras organizaciones, entre muchas otras.

- Otros múltiples cometidos informativo-documentales propios de las RR. PP.

Hay una tríada de funciones principales en la comunicación de las organizaciones:

1. Función propiamente informativa en el nivel interno de la organización. 
2. Función de difusión y comunicación pública (externa).

3. Función de legitimación en la escenificación política o empresarial. Relacionada con la anterior, y en ocasiones muy visible, se refiere a las comparecencias públicas en las que no es infrecuente que aparezcan las personalidades, especialmente los políticos -incluso en sede parlamentaria- esgrimiendo y mostrando informes en papel, recortes de prensa, gráficos, fotografías... tanto en las ruedas de prensa como en los debates y otras intervenciones televisadas o públicas. Esta utilización comporta sus riesgos (ofrecer una imagen demagógica o agresiva, consecuencias negativas por hacer públicas informaciones que muchos estiman que deben permanecer al margen del debate externo...), si bien un uso limitado, prudente y reflexivo puede resultar efectivo en términos de comunicación para reforzar la autoridad del orador como persona documentada o la verdad constatable de lo que se dice.

4. Un uso inteligente y oportuno de la documentación informativa por parte de las empresas e instituciones, integrado en la estrategia general de RR.PP. diseñada, contribuye a que haya elementos de interés humano que consigan penetrar en la agenda mediática. Los gabinetes trabajan conociendo perfectamente los criterios estandarizados de interés noticioso y las rutinas de los medios.

\section{Formatos documentales y cauces y rutinas informativas de comunicación externa}

La comunicación de las empresas e instituciones se divide tradicionalmente en comunicación interna, externa y de crisis. A ese modelo nos acogemos para ver con mayor detenimiento el ámbito de la difusión pública, por ser el más relevante en un gabinete de prensa.

\section{Comunicación externa}

La comunicación externa es aquella que se produce entre la organización y sus públicos o interlocutores externos, especialmente la prensa. Hasta tal punto que se suelen equiparar las relaciones externas a las relaciones con medios informativos de manera exclusiva. Aunque suele haber una dirección encargada de las relaciones institucionales, no es menos cierto que el gabinete u oficina de prensa se relaciona también con otros públicos a los que debe, igualmente, cuidar y tratar profesionalmente.

Además de en las relaciones estrictamente informativas; la documentación, en el marco de la comunicación, juega un destacado papel en las funciones y los procesos de planificación privada y actuación pública propios del protocolo e incluso el ceremonial. Existen necesidades de preparación, verificación y jerarquización conforme a las reglas protocolarias de tratamientos, prevalencia y precedencias en los actos públicos. Ello conlleva el manejo documental de los decretos y la legislación nacional y autonómica correspondiente así como la fijación del orden de situación en mesas y presidencias, el orden de las intervenciones y similares cuestiones.

Una política eficaz de comunicación debe cumplir ciertos requisitos básicos que las fuentes institucionales y empresariales han de tener en consideración si quieren contar con mayores garantías respecto a sus estrategias para ser seleccionadas y que se conozcan sus mensajes. Entre ellos, depender del máximo órgano de dirección dentro de la organización y plantear un enfoque global de la comunicación externa. En este sentido, un buen responsable de prensa o comunicación deberá, entre otros importantes cometidos:

- Diseñar y planificar campañas informativas, contando con una meditada estrategia de emisión documental y de organización de actos. Se debe evitar ser un "cañón" que distribuye información en exceso y también funcionar como "muro de contención" de una fortaleza hermética en la que la falta de transparencia informativa conlleve las peores consecuencias en términos de imagen y en otros aspectos (conocimiento, relevancia y notoriedad social, favor del público, etcétera).

- Enviar información a los medios, escrupulosamente cuidada en fondo y forma y por los soportes idóneos en cada ocasión.

- Atender adecuadamente las demandas de los periodistas y profesionales de la comunicación.

- Conocer las rutinas de la producción informativa (procedencia y tratamiento de la información; pautas básicas profesionales; reglas no escritas del oficio en relación con el trato con las fuentes y otros aspectos relevantes; horarios concretos de trabajo y, especialmente, de edición y cierre; adaptación al soporte de cada medio, entre muchas otras cuestiones). La mayor parte de las noticias llegan a los medios de comunicación por vías rutinarias: ruedas de prensa, comunicados, actos oficiales... y por agencias y corresponsales que utilizan esas mismas vías 
con diferencia respecto a otras: contactos personales, investigaciones propias, ser testigo de sucesos relevantes, etcétera.

- Cuidar al máximo las relaciones interpersonales con los periodistas y demás actores de la comunicación (y naturalmente conocerlos, así como sus áreas de especialización, para evitar que les lleguen noticias que no son de su competencia, lo que además de restar eficacia daría una mala imagen del departamento de prensa y de la organización). Es lógico que existan recelos mutuos entre periodistas y gabinetes. Por eso, como norma, nunca se debe tratar de imponer nada. La actitud debe ser profesional, entendiendo por tal firmeza sin presión, juego limpio.

- Fijar claramente las normas que deberán seguir los medios en la cobertura de actos. Por ejemplo, en reuniones en las que se realiza una convocatoria "sólo a gráficos" con el objeto de que se puedan tomar fotografías e imágenes de televisión, únicamente unos minutos, al inicio de las mismas pero sin permitir la captación de sonido (lo que en el argot se llama "un mudo"). Al igual que los medios, con dudoso criterio ético, a veces no respetan lo establecido, al captar y difundir conversaciones sin conocimiento de sus autores en este tipo de reuniones o en otras ocasiones; los gabinetes incurren también en abusos como las llamadas "ruedas de prensa sin preguntas", una situación denunciada en reiteradas ocasiones por la Federación de Asociaciones de la Prensa de España (FAPE).

- Estar al día respecto al sesgo ideológico de cada medio, sus principales intereses, su composición accionarial, sus alianzas y estrategias.

- Saber cuáles son los criterios de "noticiabilidad" más estandarizados, tanto los clásicos como las posibles formas emergentes de acceder al interés de las publicaciones y canales audiovisuales y de la red.

Resulta necesario centralizar la supervisión de la información externa y, según el tamaño de la organización, descentralizarla en la fase de salida o difusión a los medios de comunicación. De esta manera, se evitarán errores o contradicciones, en ocasiones de gran calado. La rápida evolución del sector de la comunicación profesional, especialmente en su dimensión tecnológica, hace que los soportes tradicionales convivan con novedosas vías para la transmisión de la información periodística. Hasta hace pocos años, aunque se pudiera desde un punto de vista fáctico, resultaba impensable lo que ahora es completamente natural: que se envíen convocatorias de prensa, alertas informativas, incluso breves comunicados, por medio de mensajes cortos SMS al teléfono móvil de los periodistas. Ya hace diez años, en 2005, el Vaticano confirmó la noticia de la muerte del Papa Juan Pablo II mediante el envío de un mensaje telefónico escrito. La presidencia del Gobierno, los partidos políticos, las empresas utilizan ya con naturalidad el sistema de avisar con un mensaje corto de las previsiones e incluso novedades informativas que se podrán ampliar contactando por teléfono con los periodistas de los gabinetes o visitando el sitio web oficial para acceder a la nota de prensa íntegra, pongamos por caso. Precisamente, Internet es una herramienta básica porque permite acercar a las fuentes de todo tipo a sus audiencias, sin necesidad de la mediación profesional de los periodistas y de sus medios; y del mismo modo esa mediación tradicional, que permite servir de gran altavoz a la organización, también se facilita gracias a la enorme rapidez y a la facilidad de publicación.

Una política eficaz de comunicación externa pasa por cumplir la visión de Sócrates: el mayor éxito de la comunicación consiste precisamente en lograr ser lo que se desea parecer.

\section{Formatos documentales y cauces y rutinas de comunicación externa}

La documentación para la comunicación externa se presenta en diversos formatos, productos o actos informativos como los siguientes, que exponemos, en elaboración propia, a partir de Piñuel (1997); GarcíaJiménez (1999); Marcos-Recio, García-Jiménez y Nuño-Moral (2004); Ramírez de la Piscina (1995) y CarrilloDurán y Nuño-Moral (2010):

1. Bases de datos sobre profesionales. Su principal valor reside en su capacidad para almacenar datos capaces de agilizar las relaciones con los periodistas. En este documento aparecerán datos de carácter práctico como el medio de comunicación al que pertenece el periodista, el teléfono, el número de fax o el correo electrónico.

2. Hoja o cuaderno de peticiones. En este documento se suele incluir las solicitudes de información procedentes del exterior de la institución (tales como petición de entrevistas o declaraciones, ampliación de datos e informaciones o documentación adicional).

3. Nota de prensa. Esta figura puede verse mejorada si se añade información relacionada con posibles fuentes de información, datos sobre otros comunicados o datos que permitan una contextualización. También se denomina comunicado, aunque esta última palabra sería más 
precisa para referirse a textos más breves y con un plus de oficialidad. Toda nota de prensa naturalmente debe tener una buena redacción y presentación, en ambos casos con mentalidad y resultados periodísticos, en ningún caso propagandísticos o publicitarios, puesto que eso desmerece tanto el contenido como a la fuente, y puede bastar para que la noticia no resulte publicada si no es especialmente relevante. Debe tener dos hojas como máximo, salvo justificadas excepciones. Su información periodística ha de ser concreta (sin divagar) e incluir datos finales sobre la entidad, en caso necesario. Si es muy conocida, algunos profesionales consideran mejor no incluir esa parte. Pero no todos están de acuerdo. De hecho es muy habitual la existencia en medianas empresas e incluso grandes corporaciones de la llamada "Carta de identidad", que constituye "un documento breve que contiene la información básica sobre lo que la empresa es” (Carrillo-Durán y Nuño Moral, 2010: 130). La apariencia debe ser de objetividad, con un esfuerzo si resulta preciso para no adjetivar. Los formatos varían según el tipo de organización y la naturaleza del hecho que se comunica. Hay algunos elementos indispensables en las plantillas que se suelen usar:

- Identidad e Imagen pública (adecuado uso de logotipos, numeración de páginas, tipografía y demás elementos de identidad corporativa). Identificación clara de la fecha; del emisor, como organización (la firma individual no siempre procede. Si se incluye, se hará al final) y, en su caso, de la ciudad, si la entidad o el organismo del que parte la información lo requiere.

- Titular (como mínimo) y, en su caso, antetítulo y/o subtítulos a la manera de sumarios. Su carácter debe ser informativo, nunca literario ni interpretativo, y más breve que largo, en forma verbal activa, con redacción sencilla (sujeto y predicado) y mencionando a la entidad u organización siempre que sea procedente y no algo forzado. En todo caso, sin grandilocuencia, con datos objetivos y nunca con valoraciones más allá de las imprescindibles.

- Cuerpo de la información. Empleo de párrafos, redacción periodística (sencillez, claridad y concisión). El párrafo inicial cumple la función de la entradilla, con la información más importante a modo de resumen periodístico. Especialmente para la prensa convencional, conviene seguir aún hoy las normas clásicas de la pirámide invertida (la información más relevante al principio y los detalles al final, en orden decreciente de interés) y dar cumplimiento dentro de lo posible a las cinco o seis $\mathrm{W}$, que responden a lo más importante: quién, qué, cuándo...

- No pueden faltar los datos de contacto para poder ampliar la información o resolver posibles dudas recurriendo a los responsables de comunicación. Es especialmente importante que existan varias posibilidades (teléfono, correo electrónico, sitio web...), sobre todo si no es una entidad demasiado conocida. Si la conoce todo el mundo (un ministerio, una multinacional popular...) se puede rebajar la aportación de esa información, pero nunca eliminar (siempre habrá periodistas nuevos, otros que no tengan los teléfonos en ese momento...). Se trata de facilitar la labor de los medios para todas las situaciones o eventualidades posibles.

Respecto a las convocatorias y las notas de prensa, se debe establecer una estrategia de emisión documental, hay que jerarquizar y planificar la información que se ofrece a los medios y en qué momentos se hace. No se puede ofrecer una continuidad de notas que sature a los medios y pueda hacer que se resienta la profesionalidad y credibilidad de la institución o empresa. De la misma manera hay que elegir el formato idóneo para esa información: por ejemplo, no sería admisible convocar una rueda de prensa con unas expectativas exageradas o sobredimensionadas (y por tanto falsas) de información para luego no aportar nada. Los medios pueden pasar factura en ese momento y/o en el futuro por hacerles perder el tiempo e incluso sentirse engañados, con razón o sin ella.

Los últimos estudios (Busto Salinas, 2014) corroboran que la influencia de los gabinetes de comunicación sigue ganando peso, especialmente con la publicación de notas de prensa en un alto porcentaje, en muchas ocasiones sin modificación o contraste por parte de los medios. Se confirma que la dependencia de éstos respecto a los departamentos institucionales de prensa es muy alta, en España como en casi todos los países. Si a finales de los setenta en el caso de la prensa española las noticias publicadas con origen en un gabinete de prensa alcanzaban el $30 \%$, a principios de los noventa superaban el 50\% (Ramírez de la Piscina, 1995), una tendencia que no haría sino aumentar considerablemente en los años siguientes, con cifras cercanas al 80\% (Elías Pérez, 2003).

4. Dossier de prensa. Según la entidad y el acto, puede interesar incluir materiales como folletos, diapositivas (previendo la cuestión de los derechos de autor) y otros soportes diversos para radio y televisión en formato profesional. 
5. Resumen o recortes de prensa (clipping o press-clipping). Cauce documental interno o internoexterno, según la decisión de cada organización. Esta modalidad documental cumple una función de observatorio por la que se puede detectar las noticias relacionadas directa o indirectamente con algún asunto relevante para la organización. De esta herramienta documental se pueden extraer modos válidos de actuación y de resolución de situaciones de crisis a partir de experiencias anteriores. Se elabora ya en soporte electrónico, pero hay organizaciones cuyos directivos quieren también papel todavía, por considerarlo más manejable. Por lo general, incluye: a) el valor publicitario estimado; b) datos sobre el medio de comunicación, el espacio destinado a la información o el lugar que ocupa; c) en ciertas organizaciones, determinación del tipo de cobertura en términos de positiva, negativa o neutra. Según el tamaño de la entidad y los recursos del gabinete de comunicación, esta labor de seguimiento informativo y tratamiento documental se realiza a través de empresas externas especializadas (outsourcing). Los resúmenes de prensa cumplen sin duda una significativa función tanto informativa como documental dentro de los gabinetes de comunicación, de ahí que hayan sido objeto no sólo de consideración en este ámbito sino también de una aproximación desde el campo jurídico (Díaz Noci, 2004).

6. Rueda de prensa. La actividad que se realiza para ofrecer una rueda de prensa está relacionada en primer término con la preparación de la persona o las personas implicadas, además de la atención a cuestiones como lugar, día, hora y resto de aspectos que deben ser sometidos ineludiblemente a evaluación por parte del gabinete o la dirección de comunicación. La planificación de las posibles preguntas, la aportación de contenidos que expliquen claramente la postura de la organización y la incorporación de un dossier al diseño de la entrevista se consideran pilares básicos de un buen planteamiento comunicativo. Con el término conferencia de prensa se suele hacer alusión al mismo tipo de actividad informativa, pero en casos concretos de mayor relevancia y con un carácter oficial añadido.

7. Convocatoria de prensa. Es el documento mediante el cual se cita a los medios de comunicación para la cobertura del acto informativo que domina buena parte de la agenda de trabajo de esos mismos medios: las ruedas de prensa. Su característica esencial ha de ser la claridad respecto a la fecha, hora, lugar, intervinientes y tema principal y, en su caso, secundarios, que motivan esa comparecencia informativa. Debe ser breve, no más de seis u ocho líneas de texto, si bien en ocasiones interesa hacer llegar a las redacciones un formato documental híbrido, al incorporar alguna información mayor en la convocatoria, lo que la asemeja a la nota de prensa. De esta manera, hay convocatorias de cierta amplitud que cubren una doble función, puesto que son publicadas o emitidas como adelanto cumpliendo la función de la tradicional nota informativa previa a lo que depare el desarrollo de la rueda de prensa.

8. Entrevista. El objetivo es conocer opiniones de expertos o responsables públicos o privados, ahondar en el perfil de alguien... La gestiona y "negocia" el gabinete de forma personal o a petición (por la guía de expertos, entrevistas previas, hechos ocurridos...). Se trata de una acción que tiene lugar dentro de la relación habitual entre los periodistas y las instituciones. Supone una señal de profesionalidad, y en concreto de habilidad y solvencia documental, entregar al periodista aquellas informaciones que le puedan ser de utilidad, tales como la biografía de la persona que va a ser entrevistada, diferentes publicaciones de y sobre el partido político, fotografías y gráficos, datos relacionados con algunos de los temas que se pueden tratar, etcétera. Por otro lado, el profesional del gabinete de comunicación tiene que procurar que el responsable entrevistado se prepare a partir de documentos elaborados al efecto, en los que se incluyan datos sobre el programa, el medio o el periodista en cuestión, sobre los contenidos que expliquen y profundicen en los asuntos que van a ser objeto de preguntas, así como datos de carácter práctico como la duración o incluso cuestiones técnicas. Hay que pensar en las posibles preguntas y planificar las mejores respuestas.

9. Publicaciones empresariales o institucionales: anuarios, informes con diversa periodicidad, libros, estudios, etcétera.

10. Discurso público: se trata de un cauce de uso frecuente también para la comunicación externa, ya sean entidades públicas o privadas (discursos como tales, conferencias, presentaciones e intervenciones de otro tipo...). Por lo general, y según el tipo de discurso que sea, se realiza parcial o totalmente por el equipo de comunicación y documentación. Esos textos suelen, y 
deben, estar muy preparados y cuidados, así como suficientemente revisados por quien los firma y/o pronuncia.

11. Guía de expertos: directorio de contactos con su área de conocimiento y líneas correspondientes de investigación para que los periodistas puedan solicitar su opinión o juicio profesional a especialistas universitarios, institucionales o de empresa.

12. Campañas especiales de publicidad:

-De productos o servicios.

-Corporativas, para crear o reforzar la imagen de marca.

De dos clases principales: institucionales-políticas o comerciales-privadas.

13. Internet: sede web corporativa. Una herramienta indispensable, debe estar actualizada de manera suficiente en función del tipo de organización y contar con el diseño, la accesibilidad y la usabilidad adecuadas, así como con los recursos documentales multimedia oportunos.

14. Folletos, cartelería, carpetas y productos de mercadotecnia.

Es también frecuente que se acuerden comidas y se lleven a cabo otro tipo de contactos personales entre responsables de gabinetes y de medios. El porcentaje es muy alto y de su éxito depende llegar a otras formas de difusión como el patrocinio, el mecenazgo, la firma de convenios marco y convenios específicos de colaboración, etcétera. Hay una hibridación de información y publicidad en muchos de los formatos y cauces referidos, una realidad a la que no escapan hoy algunos de los contenidos de los propios medios. El gabinete de prensa debe procurar que el desayuno, la firma, presentación, rueda de prensa, etcétera sea un acto independiente de aquel dirigido a públicos generales o más amplios, para que la prensa pueda hacer bien su trabajo sin que eso incomode a los asistentes al acto general. En ocasiones, los directivos quieren hacer un acto único con dimensión tanto institucional como informativo-periodística, lo que, salvo excepciones, constituye un error que puede deslucir y restar eficacia a ambos aspectos del evento. Es necesaria la preparación previa exhaustiva (composición de la mesa, secuencia o programación del acto, dossier para los medios o adecuada infraestructura). Por ejemplo, para la planificación de una entrevista radiofónica solicitada, el técnico de comunicación de un gabinete, de cara a la preparación interna de los mensajes y las formas, deberá conocer varios aspectos: por supuesto el medio, sección y/o programa; si es en directo o en diferido (grabada); individual o grupal (en el último caso, qué personas o al menos qué instituciones o empresas participan); quién entrevista; sobre qué temas (más o menos); tipo de preguntas ("normales" o batería de preguntas cortas de carácter personal a la manera del llamado Cuestionario de Proust...); en qué programa se encuadra (tono, intención...); la duración; si presentarán nuestro proyecto o empresa o debe hacerlo, en su caso, el invitado; qué otros entrevistados anteriores ha habido; el perfil de la audiencia... Naturalmente se trata de saber lo máximo posible para preparar la intervención y saber de qué tipo ha de ser en función del marco o contexto general del programa, pero sin agobiar a productores y periodistas ni someterlos a ningún "tercer grado" improcedente. De parte de ésas y otras preguntas ya conoceremos la respuesta y, además, nos interesará dominar unos aspectos en unas situaciones y otros en momentos diferentes. Pero sin dejar nunca la intervención al mero azar o la información necesaria sin conocer.

\section{A modo de conclusión}

Las funciones de la documentación en el seno de los gabinetes de comunicación gestionados dentro de las organizaciones empresariales e institucionales son amplias y significativas. La evolución experimentada dentro de las Relaciones Públicas, desde los primeros desarrollos en Estados Unidos hasta la situación actual en todos los países del mundo desarrollado, ha concedido un protagonismo creciente a este tipo de fuentes informativas, cuyo trabajo no puede resultar exitoso sin una adecuada planificación y gestión documental. Dicha gestión combina los cauces y formatos más tradicionales con los nuevos soportes tecnológicos para la difusión informativa.

Son muchas las voces desde el ámbito del Periodismo que consideran, con razón, que existe una excesiva permeabilidad e incluso seguidismo de las fuentes empresariales e institucionales representadas por los 
gabinetes de prensa. De este modo tales organizaciones consiguen imponer su discurso e intereses con relativa facilidad, mediante la publicación íntegra en muchas ocasiones de sus notas de prensa, lo que disminuye la cantidad y e incluso la calidad del trabajo informativo de producción propia.

El trato habitual con los medios de comunicación (dimensión externa) es la principal tarea del gabinete de prensa, aunque no conviene descuidar tampoco los procesos informativos y documentales internos, así como el aspecto crucial de contar con una suficiente y adecuada documentación en relación con eventuales situaciones de crisis.

\section{Bibliografía}

BUSTO SALINAS, L. (2014). "Trascendencia de los gabinetes de comunicación en la práctica periodística. El caso concreto de la nota de prensa". Historia y Comunicación Social, v. 18, pp. 601-612.

CARRILLO DURÁN, M. V. y NUÑO MORAL, M. V. (2010). "La documentación en la evaluación y gestión de la imagen corporativa”. El profesional de la información, marzo-abril, v. 19, n. 2, pp. 123-132.

DÍAZ NOCI, J. (2004). Los resúmenes de prensa en los gabinetes de comunicación: una aproximación jurídica. La Coruña: Netbiblo.

ELÍAS PÉREZ, C. (2003). "Adaptación de la metodología de "observación participante" al estudio de los gabinetes de prensa como fuentes periodísticas". EMPIRIA, Revista de metodología de las Ciencias Sociales, núm. 6, pp. 145-159.

GARCÍA-GUTIÉRREZ, A. y MARTíNEZ-ÁVILA, D. (2014). "Formación crítica de documentalistas en medios de comunicación". El profesional de la información, septiembre-octubre, v. 23, n. 5, pp. 493-500.

GARCíA-JIMÉNEZ, A. (1999). "La Documentación de la Publicidad y de las Relaciones Públicas". En: García Gutiérrez, A. (ed.). Introducción a la Documentación Informativa y Periodística. Sevilla: Mad. Pp. 487-509.

JOLY, M. Diálogo en el infierno entre Maquiavelo y Montesquieu (2002). Barcelona: El Aleph Editores. LÓPEZ YEPES, J. (2011). La sociedad de la documentación. Madrid: Fragua.

MARCOS RECIO, J. C., GARCÍA JIMÉNEZ, A. y NUÑO MORAL, M. V. (2004). Gestión de la documentación en la Publicidad y en las Relaciones Públicas. Madrid: Síntesis.

MARTíN, F. (2006). Comunicación empresarial e institucional. Madrid: Universitas.

PIÑUEL, J. L. (1997). Teoría de la Comunicación y gestión de las organizaciones. Madrid: Síntesis.RAMíREZ DE LA PISCINA, T. (1995). Gabinetes de comunicación. Funciones, disfunciones e incidencia. Barcelona: Bosch.

\section{Datos del autor}




\section{Juan-Francisco Torregrosa Carmona}

Es profesor titular del área de conocimiento de Periodismo en la Universidad Rey Juan Carlos, en la que trabaja desde 2004 a tiempo completo. Doctor por la Complutense (Ciencias de la Información, sobresaliente cum laude por unanimidad), donde fue personal docente e investigador. Licenciado en Periodismo y Licenciado en Derecho. Entre 1996 y 2004, trabajó como periodista y documentalista en medios de comunicación públicos y privados, y en la Administración Pública. Ha impartido clase en programas de doctorado y en títulos de máster de la Agencia EFE, Radio Televisión Española (RTVE) y otras instituciones y universidades tanto españolas como internacionales. Dentro de estas últimas ha sido conferenciante e investigador invitado en varios países de Europa, América y Asia (Waseda, en Tokio, y Beijing Language and Culture University (BLCU), de Pekín, entre otras).

juanfrancisco.torregrosa@urjc.es

Recibido - Received : 2014-10-30

Aceptado - Accepted: 2014-12-30

\section{$(\mathrm{cc}) \mathrm{BY}$}

This work is licensed under a Creative Commons Attribution 4.0

United States License.

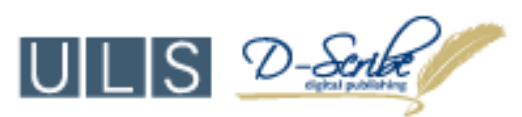

This journal is published by the University Library System of the University of Pittsburgh as part of its

D-Scribe Digital Publishing Program and is cosponsored by the University of Pittsburgh Press. 